\title{
Electrochemical Sensitive Detection of Hydrazine Through Cobalt Doped Cerium Oxide Nanostructured Platform
}

\author{
Anees AHMAD Ansari ( $\square$ aneesaansari@gmail.com ) \\ King Saud University https://orcid.org/0000-0002-8708-6673 \\ Manawwer Alam \\ King Saud University College of Science
}

\section{Research Article}

Keywords: Cerium oxide, cobalt, hydrazine, electrochemical, impedance

Posted Date: February 15th, 2021

DOl: https://doi.org/10.21203/rs.3.rs-192694/v1

License: (c) (1) This work is licensed under a Creative Commons Attribution 4.0 International License.

Read Full License

Version of Record: A version of this preprint was published at Journal of Materials Science: Materials in Electronics on April 24th, 2021. See the published version at https://doi.org/10.1007/s10854-021-059659. 


\section{Abstract}

Chemically synthesized cobalt-doped cerium oxide nanoparticles $\left(\mathrm{CeO}_{2}\right.$ :Co;NPs) were successfully prepared by complexed based co-precipitation process. The structural, morphological, chemical composition, optical properties, and electro-catalytic properties were determined by X-ray diffraction pattern(XRD), transmission electron microscopy(TEM), energy dispersive $x$-ray analysis (EDX), UV/Visible absorption spectroscopy, and cyclic voltammetry techniques. Owing to the wide-spread applications of $\mathrm{CeO}_{2} \mathrm{NPs}$ in various fields of applied material sciences, transition metal ions doped $\mathrm{CeO}_{2}: \mathrm{Co} \mathrm{NPs}$ exhibited excellent electro-catalytic properties. Outstanding physiochemical properties of $\mathrm{CeO}_{2}$ such as reversible oxidation states, high ionic mobility, large oxygen storage ability, effective large specific surface area, and the excellent current response observed in the electrocatalytic oxidation of hydrazine. The presence of transition metal ions (cobalt) improves the oxidation potential of hydrazine. Cyclic voltammetry was analog with the electrochemical impedance spectroscopy results, which revealed the enhanced with rapid sensing response against hydrazine. The electro-catalytic results of the $\mathrm{CeO}_{2}: \mathrm{Co} \mathrm{NPs}$ electrode exhibited excellent voltammetry and impedance spectroscopy performance towards the hydrazine oxidation. The fabricated chemical sensor shows a wide linear detection range from 7.18 to $1000 \mu \mathrm{M}$, a low detection limit $7.2 \mu \mathrm{M}$, and sensitivity of $2.42 \mu \mathrm{AmL} \mu \mathrm{M}^{-1} \mathrm{~cm}^{-2}$. The fabricated sensing electrode demonstrated long-term steadiness resulting it shows high sensitivity, selectivity, repeatability/reproducibility, and rapid detection of hydrazine.

\section{Introduction}

Hydrazine is widely used in various fields of applied industries including fuel cells, rocket engineering, pharmaceuticals, and various corrosive inhibitor chemicals[1,2]. It is highly volatile, toxic, and can be absorbed easily orally, through dermal or inhalation ways $[3,4]$. Furthermore, hydrazine is also identified as a mutagenic \& carcinogenic substance and can damage the lungs, liver, kidneys, and nervous system[5]. Additionally, because of the high absorption ability of hydrazine, its severe contact to the humane body leading to death[6]. Owing to their highly dangerous and environmental harmfulness draw attention to sensitive and selective detection of hydrazine. Previously, hydrazine has been determining by various techniques such as chromatographically, chemiluminescence, and spectrophotometrically, but most of them suffering from sensitivity, selectivity, reproducibility, time-consuming, expensive, and complicated detection method[1]. Therefore, the detection of hydrazine by alternate technique is an urgent need in the current era. Currently, the electrochemical technique has been considered the most effective, sensitive, accurate, very simple, and efficiently detect various analytes[7-15]. Because of its simplicity and accurate detection, reproducible results have been effectively applying in the detection of chemically or non-chemically hazardous species. Although, some analytes have high redox potential with a lower current over the surface of the non-fabricated electrode. Therefore, the overvoltage of the reaction chemically changed electrodes increase the rate of electron transfer reaction. In that case, the oxidation of hydrazine on the surface of the glassy carbon electrode (GCE) has a high excess potential and an 
exceedingly low oxidation current. Although hydrazine oxidation can easily be carried out on indium-tinoxide, Au, and Pt electrodes, later two metals are very costly.

Recently, semiconductor metal oxides have been extensively applied either in catalysts, photocatalysts, fuel cells, gas sensors, chemical sensors, UV/visible blocking agents, and in polishing materials[9, 11-13, 16]. It has been recognized that the crystal planes exposed to semiconductor metal oxides that define the surface arrangement and structure of the surface have a significant effect on their exterior reactivity and electro-catalytic efficiency. Among the various semiconductor metal oxides, cerium oxide has been considered the most fascinating semiconductor metal oxide, because its unique physiochemical properties make them differ from the rest of the semiconductor metal oxides[17-20]. $\mathrm{CeO}_{2}$ demonstrate reversible oxidation states, resulting it exhibits redox characteristic and high oxygen storage ability $[17,19$, 20]. These novel properties raise their technological applications in extensive fields such as gas sensors, catalysts, photo-catalysts, capacitors, hydro-splitting, solid fuel cells, UV resistance, and polishing materials. Liu et al. successfully applied $\mathrm{Co} / \mathrm{Ni}$ binary doped $\mathrm{CeO}_{2}$ mesoporous nanospheres for $\mathrm{CO}$ catalytic oxidation[21]. Zhang et al observed excellent $\mathrm{CO}$ catalytic properties of the $\mathrm{Cu}$ ion-doped ceria NPs[22]. Kumar et al observed enhance bandgap energy on doping of Mn ion in ceria film[23]. Malhotra and his associates have been successfully applied pure sol-gel prepared films for the detection of various anlytes $[9,10,13,14]$. It has been well-accepted that the doping of impurities in the host lattices disbalance the crystal structure and altered the crystal growth as well as lattice parameters[24]. The disturbance of the lattice parameters enhanced the oxygen ion mobility within the lattice, resulting in improve conducting properties of the semiconductor metal oxides[25, 26]. Therefore, the insertion of impurities within the metal oxide lattices has been demonstrated as an excellent process to promote the physiochemical properties of the semiconductor metal oxides. So that, on integration of the transition metal as a dopands within the ceria crystal lattice, the high movement of oxygen vacancies, outstanding redox performance, large specific surface area, lattice defects and synergistic effects can be enhanced. The inclusion of ceria crystal lattice impurities improved the ionic mobility within the crystal lattice, increasing the potential of ionic conductivity or ion release. Nevertheless, $\mathrm{CeO}_{2}$ lattice mobility of ionic species depends on the form of dopant ion, the amount of dopant and the concentration of ionic species. Furthermore, due to their charge compensation within the lattice, the dopant ions can dramatically upsurge the number of ionic compounds, resulting in high electro-oxidation of the redox species' properties. Recently some efforts have shown that the addition of transition metal ions impurities remarkably enhanced the conducting as well as magnetic properties as prepared by chemical routes [17$20,27]$.

In this manuscript, we synthesized cobalt doped $\mathrm{CeO}_{2}$ nanoparticles by a simple co-precipitation process. Ethylene glycol was used as a chelating agent to control the mobility or growth of the nucleons during the hydrolysis of cerium nitrate in the presence of an ammonia base. The preparation $\mathrm{CeO}_{2}$ : $\mathrm{Co} \mathrm{NPs} \mathrm{were}$ successfully utilized in electrochemical sensor development. The crystallographic structure, morphology, and optical properties were monitored by X-ray diffraction(XRD), field emission transmission electron microscopy(FE-TEM), and UV/visible absorption spectroscopy techniques. Energy dispersive analysis 
was verified the chemical composition of the as-prepared material. Electrochemical properties were determined by cyclic voltammetry and electrochemical impedance spectroscopy techniques. The developed chemical sensor is highly sensitive for lower range detection of hydrazine concentration. Here, we applied chemical homogeneous co-precipitation route for synthesis of ceria nanoparticles because of its simplicity, eco-friendly, low cost, easy processing technique.

\section{Experimental \\ 2.1. Materials}

Cerium nitrate hexahydrate (99.9\% BDH chemicals, England), cobalt nitrate (E-Merck, Germany), hydrazine (99.9\% Sigma-Aldrich, USA), ethylene glycol, and ammonia were analytical grade used directly as a starting material without further purification. Millipore (Milli-Q, Bedford, USA) water was used for the synthesis and characterization of the samples.

\subsection{Synthesis}

For the synthesis of cobalt doped cerium oxide NPs, $0.93 \mathrm{~mol}(2 \mathrm{M})$ cerium nitrate and $0.07 \mathrm{~mol}(2 \mathrm{M})$ cobalt nitrate were dissolved separately in Milli-Q water and later mixed dropwise in $50 \mathrm{ml}$ ethylene glycol on a hot plate with constant mechanical stirring. After homogenous mixing, a transparent solution was obtained $[9,13,15]$. In this homogenous mixed solution, ammonia solution was added dropwise with magnetic stirring for decomposition. In addition to the ammonia solution a dirty grey color precipitate appeared. An obtained precipitate was separated by centrifuged, and washed several times with water to remove the excess amount of unreacted reactants, and dried at $100^{\circ} \mathrm{C}$. Later this power was dried in an oven at $300^{\circ} \mathrm{C}$ for complete conversion into metal oxide.

\subsection{Characterization}

X-ray diffraction pattern (PANalytical X Part, X-ray diffractometer) equipped with Ni filter Cu Ka ( $\lambda=$ $1.5404 \AA$ A ) radiation. Field Emission-Transmission electron microscopes (FE-TEM, JEOL, JEM-2100F, Japan) equipped with energy dispersive $x$-ray analysis(EDX) were utilized for monitoring the morphological structure and chemical composition operating at an accelerating voltage $2000 \mathrm{kV}$. UV/Visible spectrum was measured from Cary 2000(Agilent Technologies, USA) spectrophotometer within the 200-800 nm spectral range. Three electrode systems of Metrohm Autolab analyzer (PGSTA30, Switzerland) were applied for the determination of electrochemical characteristics of the nanomaterial. In this three-electrode arrangement, the working electrode was modified from $\mathrm{CeO}_{2}: \mathrm{Co} / \mathrm{glassy}$ carbon electrode(GCE), platinum(Pt) as a counter electrode, and $\mathrm{Ag} / \mathrm{AgCl}$ was used as a reference electrode. All these electrochemical experiments were conducted in environmental conditions.

\section{Results And Discussion}

3.1. Crystallographic structure 
Crystallographic structure, phase purity, and crystallinity was determined from X-ray diffraction pattern. Figure 1 displays the main reflection peaks assigned to (111), (200), (220), (311), (222), and (400), which are well-matched and identified to the pure cubic fluorite structure in according to the standard data[2830]. It is obvious from the reflection planes the widths of lines are broader, signifying that the product is very small in size with well crystalline. The intensities and peak positions of the diffraction planes are well-suited with the literature reports[17, 18]. Over the entire range of XRD pattern, no reflection planes of cobalt oxide were observed it the indicate that the Co ions are homogeneously distributed within the ceria lattice, which forms the Ce-O-Co solid solution. Average crystalline size was calculated from the halfwidth of well-dominated (111) reflection planes via the Sherrer equation was to be $10 \mathrm{~nm}[17,18,20]$.

\subsection{Morphological shape}

Morphological images of the as-synthesized nanoproduct were obtained from FE-TEM micrographs to inspect the shape and size. Figure $2 \mathrm{a} \& \mathrm{~b}$ demonstrates the irregular quasi-spherical shaped, rough surface, highly aggregated, narrow size distributed, highly crystalline nanostructures with crystalline size about $5-8 \mathrm{~nm}$. The nanoproduct is highly aggregated because of the small grain size with high surface area, which was prepared in aqueous media at environmental condition, so that may be little amount organic moiety still attached over the surface of nanoparticles. As observed in TEM images Fig. $2 \mathrm{~d} \&$, in high magnifying images well-defined individual lattice fringes are apparent at a distance of about 0.29 $\mathrm{nm}$, which are fully ascribed to the (111) lattice plane of a fluorite cubic shapes crystal structure[17, 18]. Additionally, the crystallinity of the nanoparticles was further inspected by selected area electron diffraction (SAED) analysis. The SAED pattern in Fig. 2c illustrates the four broad ring structures with dspacing of around $0.30 \mathrm{~nm}$, which endorsed to (111), (200), (220), and (311) diffraction plane of the cubic-fluorite structure. The EDX analysis in Fig. $2 f$ verified the existence of all expected elements such as Cerium (Ce), Cobalt (Co), and oxygen ( 0 ). It advocated that $\mathrm{Co}$ ions are homogeneously distributed inside the $\mathrm{CeO}_{2}$ : $\mathrm{Co}$ crystal lattice. Additionally, the absence of any impurity peaks in EDX spectrum illustrates the phase purity of the nanoproduct. The existence of strong peaks of $\mathrm{C}$ and $\mathrm{Cu}$ are belonging to the carbon-coated copper grids (Fig. 3f).

\subsection{Optical properties}

UV/Visible spectrum was measured to examine the optical properties as illustrated in Fig. 3a. The optical absorption spectrum in Fig. 3a displays a broad with the high-intensity band in the UV/visible range originating from the charge transfer amid the oxygen to $\mathrm{Ce}^{3+/ 4+}$ ion. The energy bandgap $\left(E_{\mathrm{g}}\right)$ value was determined from the linear portion of the optical absorption spectrum through Tauc and Menths formulae[28, 29, 31, 32]. The $E_{\mathrm{g}}$ value based on the equation was estimated at $3.0 \mathrm{eV}$ (Fig. 3b), which is closely matched with the published literature reports[9, 10, 14].

\subsection{Electrochemical properties}

Cyclic voltammetry studies were carried out to investigate the electrochemical properties of the asdesigned cobalt ions doped $\mathrm{CeO}_{2}$ electrode monitored in phosphate buffer solution(PBS) for detection of hydrazine. Figure 4a illustrates the comparative analysis of the surface-modified electrode in similar 
potential conditions $(-1.75$ to $+1.75 \mathrm{~V})$ with a scan rate of $100 \mathrm{mVs}^{-1}$. As shown in Fig. 4, the bare GCE did not reveal any redox activities because of the absence of electrochemical behavior over the surface of the electrode. After deposition of cobalt ions doped $\mathrm{CeO}_{2} \mathrm{NPs}$ layer over the GCE, the spectrum exhibited redox peaks, which was more apparent on measuring in hydrazine. The existence of a broad oxidation peak in the spectrum shows the electrocatalytic properties of the as-designed electrode. Furthermore, it indicated that a highly crystalline $\mathrm{CeO}_{2}$ : $\mathrm{Co} / \mathrm{GCE}$ electrode facilitates fast electron communication in the presence of PBS resulting in oxidization of the hydrazine. It is well-accepted that the high oxygen storage ability of ceria remarkably accelerates the electrocatalytic performance of the fabricated electrode. Electrocatalytic performance of the fabricated electrode $\left(\mathrm{CeO}_{2}: \mathrm{Co} / \mathrm{GCE}\right)$ was measured at different scan rates by cyclic voltammetry as demonstrated in Fig. 4b\&c. As displayed in Fig. 4, the redox peak was significantly shifted towards the positive side along with peak-to-peak separation on increasing the scan rate that is a validation of oxidation process in diffused controlled[13, 14]. Furthermore, it suggested that the redox behavior of ceria and reversible valence with high oxygen storage ability facilitate electrocatalytic performance. An observed linear increment in the alteration amid cathodic $\left(E_{\mathrm{pc}}\right)$ and anodic $\operatorname{peak}\left(E_{\mathrm{pa}}\right)$ shift $\left(\Delta E=E_{\mathrm{pa}}-E_{\mathrm{pc}}\right)$ demonstrated the chemical kinetic of electron communication over the surface of the fabricated electrode[8, 14].

\subsection{Electrochemical sensing by cyclic voltammetry}

Figure $5 \mathrm{a}$ illustrates the results of hydrazine sensing by using the electrochemical method as a function of hydrazine concentration at constant electrode potential. As represented in the spectra the electrochemical performance was measured under the potential range from -1.75 to $1.75 \mathrm{~V}$ at $100 \mathrm{mV}$ current. As observed in Fig. 5a, the magnitude of peak redox potential was significantly enhancing linearly along with increasing hydrazine concentration within the range of 7.185-1000 $\mu \mathrm{M}$ in $100 \mathrm{~mL}$ PBS. The redox peak current was gradually increased on increasing the hydrazine concentration, it advocated that the current in the form of peak position was up surging with the increase in hydrazine concentration that parallels the rapid relocation of electrons at the conduction band. Figure $5 \mathrm{~b}$ revealed the sensing parameters, which were obtained from the electrochemical redox peak results. The fabricated sensing electrode exhibited a linear detection range from 7.185 to $1000 \mu \mathrm{M}$ with high sensitivity 2.42 $\mu \mathrm{AmL} \mu \mathrm{M}^{-1} \mathrm{~cm}^{-2}$ and detection limit(LOD) was $7.2 \mu \mathrm{M}$. As represented in the graph the redox peak current was remarkably rising on increasing high concentration range of hydrazine in the PBS solution, it implies that the fabricated electrode is more efficient to catalyze the hydrazine as well as fast detection of the analyte.

\subsection{Electrochemical sensing by electrochemical impedance spectroscopy}

The sensing performance of the as-designed electrode was further examined by the electrochemical impedance spectroscopy method, which is the most effective technique to investigate the electrocatalytic processes over the surface of $\mathrm{CeO}_{2}: \mathrm{Co} / \mathrm{GCE}$ electrode. As observed in Fig. 6, the Nyquist plot in EIS spectra demonstrates the semicircle structure that is represented to the electron transfer resistance 
and directed by the redox-probe electron communication kinetics at the electrode surface[7, 12, 28]. The variation in semicircle shape represents the conducting efficiency of the probe, as the surface is covered or adsorbed by conducting/semiconducting or insulating molecules/layer suppressed or upsurge the semicircle shape resulting electron-transporting values varies. In Fig. 6, we measured the resisting/conducting efficiency of the fabricated $\left(\mathrm{CeO}_{2}: \mathrm{Co} / \mathrm{GCE}\right)$ electrode at different hydrazine concentration. In a comparative analysis, the semicircle shape was reduced as increase the hydrazine concentration, which implies that the fabricated $\mathrm{CeO}_{2}: \mathrm{Co} / \mathrm{GCE}$ electrode accelerates the electron transfer rate over the electrode surface. Furthermore, hydrazine is known as a high oxidizing agent, and the presence of fabricated $\mathrm{CeO}_{2}: \mathrm{Co} / \mathrm{GCE}$ electrode speedup the diffusion of electrons towards the electrode surface resulting in altered the semicircle shape or electron transfer rate. On induction of hydrazine, the observed change in the electron transfer rate can be assigned to the dielectric and conducting characteristics at the electrode-electrolyte interface consistent with the successive oxidation of hydrazine molecules. These findings demonstrated that the fabricated $\mathrm{CeO}_{2}: \mathrm{Co} / \mathrm{GCE}$ electrode creates excellent electron pathways between electrolytes and electrodes and can be used as a good platform for electrochemical sensing.

\subsection{Electrochemical stability of the sensing electrode}

The electrochemical stability or reproducibility of the as-designed $\mathrm{CeO}_{2}: \mathrm{Co} / \mathrm{GCE}$ electrode was examined by the cyclic voltammetry technique at ten successive cycles in the presence of hydrazine $(500 \mu \mathrm{M})$ in 1.0 mM PBS. As shown in Fig. 7 the fabricated electrode exhibited excellent stability and reproducibility, it endorsed that the fabricated sensing electrode is suitably reproducible. These findings revealed that, because of its excellent durability and reproducible performance, the developed $\mathrm{CeO}_{2}: \mathrm{Co} / \mathrm{GCE}$ sensing electrode is highly appreciable for generating the sensing results.

\section{Conclusion}

Chemically synthesized $\mathrm{CeO}_{2}$ :Co NPs prepared by co-precipitation process were deposited on GCE to examined the sensing properties of the as-prepared NPs against hydrazine through a three-electrode system. XRD and TEM results exhibited that the prepared NPs are small having 8-10 nm in size, with an irregular spherical shape. Absorption spectra were verified the large band gap $(\sim 3.0 \mathrm{eV})$ with strong absorption in the visible range. The $\mathrm{CeO}_{2}$ : $\mathrm{Co}$ NPs fabricated electrode revealed excellent electrocatalytic performance in PBS solution, which was remarkably improved in the presence of hydrazine. The cyclic voltammetry and impedance spectroscopy result revealed the rapid ion-exchange process in the buffer solution. A large concentration range of hydrazine from 7.18 to $1000 \mu \mathrm{M}$ was applied to determine the sensing activity of the fabricated electrode. In sensitivity results, the redox peaks are progressively altered on adding the hydrazine concentration, which was remarkably enhanced on enhancing the concentration quantity, it represented that the fabricated electrode is highly sensitive with specific and sensitive detection of hydrazine. EIS sensitivity results were validated the cyclic voltammetry results and suggested that the fabricated electrode is highly sensitive for the detection of hydrazine concentrations. The fabricated electrode shows high stability which is sufficient sustainability for a longer period. 


\section{Declarations}

Acknowledgment: The authors extend their appreciation to the Deanship of Scientific Research, King Saud University, Riyadh, for funding this work through Research Group No. RG -1439-089.

Declaration: The authors declare no competing financial or personal relationship conflict of interest.

\section{References}

[1] A Benvidi, S Jahanbani, B-F Mirjalili, R Zare (2016) Chinese Journal of Catalysis 37: 549. Doi:https://doi.org/10.1016/S1872-2067(15)61046-4

[2] J Zhang, W Gao, M Dou, et al. (2015) Analyst 140: 1686. Doi:10.1039/C4AN02111H

[3] R Devasenathipathy, V Mani, S-M Chen (2014) Talanta 124: 43.

Doi:https://doi.org/10.1016/j.talanta.2014.02.031

[4] K Yamada, K Yasuda, N Fujiwara, et al. (2003) Electrochemistry Communications 5: 892. Doi:https://doi.org/10.1016/j.elecom.2003.08.015

[5] H Ahmar, S Keshipour, H Hosseini, AR Fakhari, A Shaabani, A Bagheri (2013) Journal of Electroanalytical Chemistry 690: 96. Doi:https://doi.org/10.1016/j.jelechem.2012.11.031

[6] L Cui, Z Peng, C Ji, et al. (2014) Chemical Communications 50: 1485. Doi:10.1039/C3CC48304E

[7] AA Ansari, A Kaushik, PR Solanki, BD Malhotra (2009) Electroanalysis 21: 965.

Doi:10.1002/elan.200804499

[8] AA Ansari, PR Solanki, BD Malhotra (2009) Journal of Biotechnology 142: 179.

Doi:10.1016/j.jbiotec.2009.04.005

[9] PR Solanki, C Dhand, A Kaushik, AA Ansari, KN Sood, BD Malhotra (2009) Sensors and Actuators BChemical 141: 551. Doi:10.1016/j.snb.2009.05.034

[10] A Kaushik, PR Solanki, AA Ansari, S Ahmad, BD Malhotra (2009) Nanotechnology 20. Doi:Artn 055105

$10.1088 / 0957-4484 / 20 / 5 / 055105$

[11] A Ali, AA Ansari, A Kaushik, et al. (2009) Materials Letters 63: 2473.

Doi:10.1016/j.matlet.2009.08.038

[12] AA Ansari, A Kaushik, PR Solanki, BD Malhotra (2010) Bioelectrochemistry 77: 75.

Doi:10.1016/j.bioelechem.2009.06.014 
[13] AA Ansari, A Kaushik, PR Solanki, BD Malhotra (2008) Electrochemistry Communications 10: 1246. Doi:10.1016/j.elecom.2008.06.003

[14] AA Ansari, PR Solanki, BD Malhotra (2008) Applied Physics Letters 92. Doi:Artn 263901

$10.1063 / 1.2953686$

[15] AA Ansari, PR Solanki, BD Malhotra (2009) Sensor Letters 7: 64. Doi:10.1166/sl.2009.1011

[16] K Gadani, MJ Keshvani, B Rajyaguru, et al. (2017) Physical Chemistry Chemical Physics 19: 29294. Doi:10.1039/c7cp03187d

[17] AA Ansari, J Labis, M Alam, SM Ramay, N Ahmad, A Mahmood (2016) Journal of Electroceramics 36: 150. Doi:10.1007/s10832-016-0018-1

[18] AA Ansari, J Labis, M Alam, SM Ramay, N Ahmad, A Mahmood (2015) Journal of the Chinese Chemical Society 62: 925. Doi:10.1002/jccs.201500195

[19] AA Ansari, JP Labis, M Alam, SM Ramay, N Ahmed, A Mahmood (2017) Analytical Letters 50: 1360. Doi:10.1080/00032719.2016.1218499

[20] AA Ansari, JP Labis, M Alam, SM Ramay, N Ahmad, A Mahmood (2016) Acta Metallurgica SinicaEnglish Letters 29: 265. Doi:10.1007/s40195-016-0387-0

[21] X Liu, L Han, W Liu, Y Yang (2014) European Journal of Inorganic Chemistry 2014: 5370.

[22] J Zhang, J Guo, W Liu, et al. (2015) European Journal of Inorganic Chemistry 2015: 969.

[23] CSS Pavan Kumar, R Pandeeswari, BG Jeyaprakash (2014) Journal of Alloys and Compounds 602: 180. Doi:https://doi.org/10.1016/j.jallcom.2014.02.143

[24] S Maensiri, C Masingboon, P Laokul, et al. (2007) Crystal Growth \& Design 7: 950.

Doi: $10.1021 / \mathrm{cg} 0608864$

[25] C Xia, C Hu, P Chen, B Wan, X He, Y Tian (2010) Materials Research Bulletin 45: 794.

Doi:https://doi.org/10.1016/j.materresbull.2010.03.015

[26] RD Kerkar, AV Salker (2020) Materials Chemistry and Physics 253: 123326.

Doi:https://doi.org/10.1016/j.matchemphys.2020.123326

[27] SM Ramay, A Mahmood, S Atiq, AN Al Hazaa (2016) International Journal of Modern Physics B 30. Doi:Artn 1650020

$10.1142 / \mathrm{S} 021797921650020 x$ 
[28] HA Hussain, AA Ansari, K Iftikhar (2004) Spectrochimica Acta Part a-Molecular and Biomolecular Spectroscopy 60: 873. Doi:10.1016/S1386-1425(03)00312-3

[29] AA Ansari, SP Singh, BD Malhotra (2011) Journal of Alloys and Compounds 509: 262.

Doi:10.1016/j.jallcom.2010.07.009

[30] AA Ansari, M Azahar, BD Malhotra (2012) India-Japan Workshop on Biomolecular Electronics and Organic Nanotechnology for Environment Preservation 358. Doi:Artn 012006

$10.1088 / 1742-6596 / 358 / 1 / 012006$

[31] AA Ansari, A Kaushik (2010) Journal of Semiconductors 31: 033001. Doi:10.1088/16744926/31/3/033001

[32] AA Ansari (2010) Journal of Semiconductors 31: 053001. Doi:10.1088/1674-4926/31/5/053001

\section{Figures}




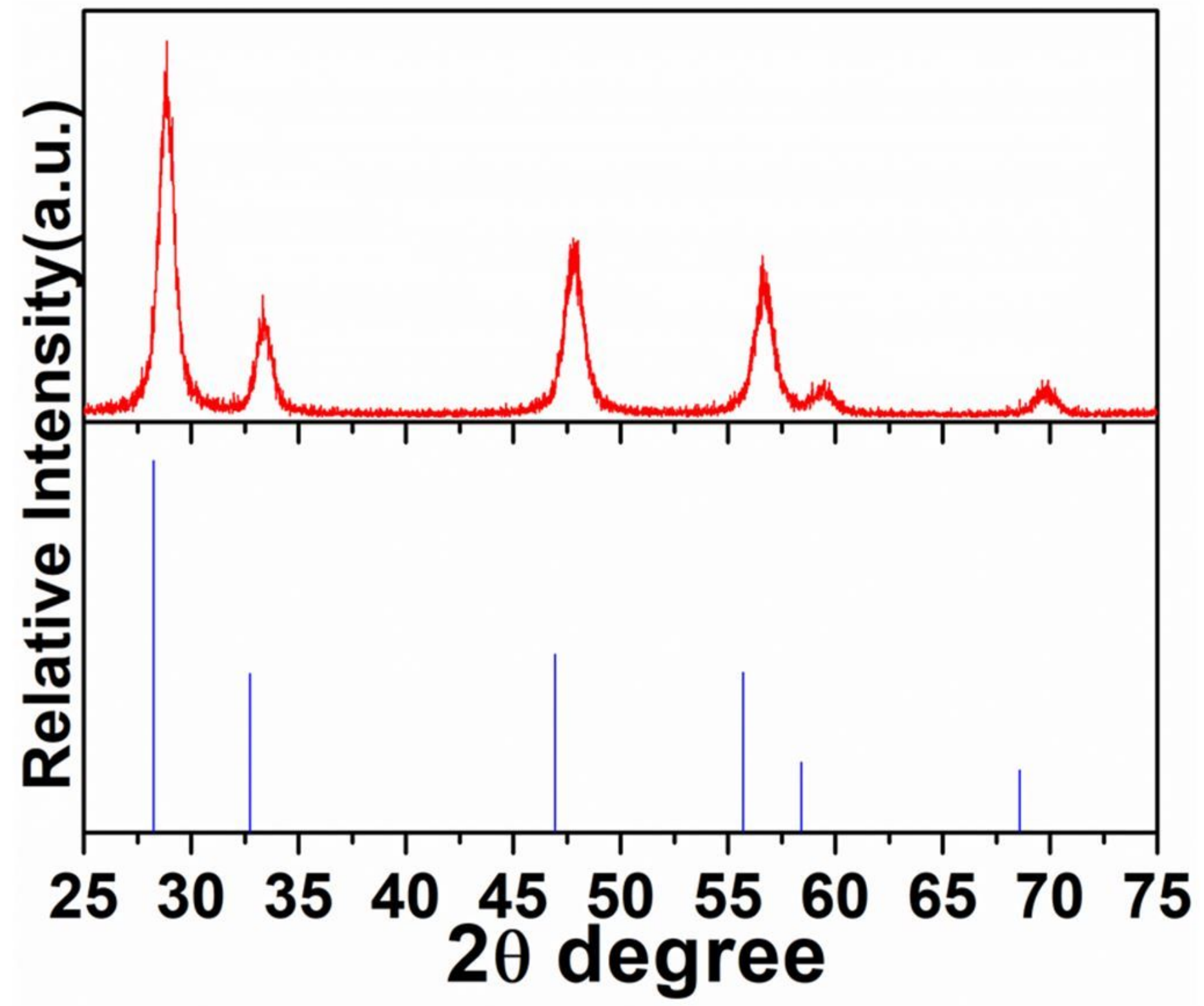

Figure 1

X-ray diffraction pattern of $\mathrm{CeO} 2$ :Co NPs. 

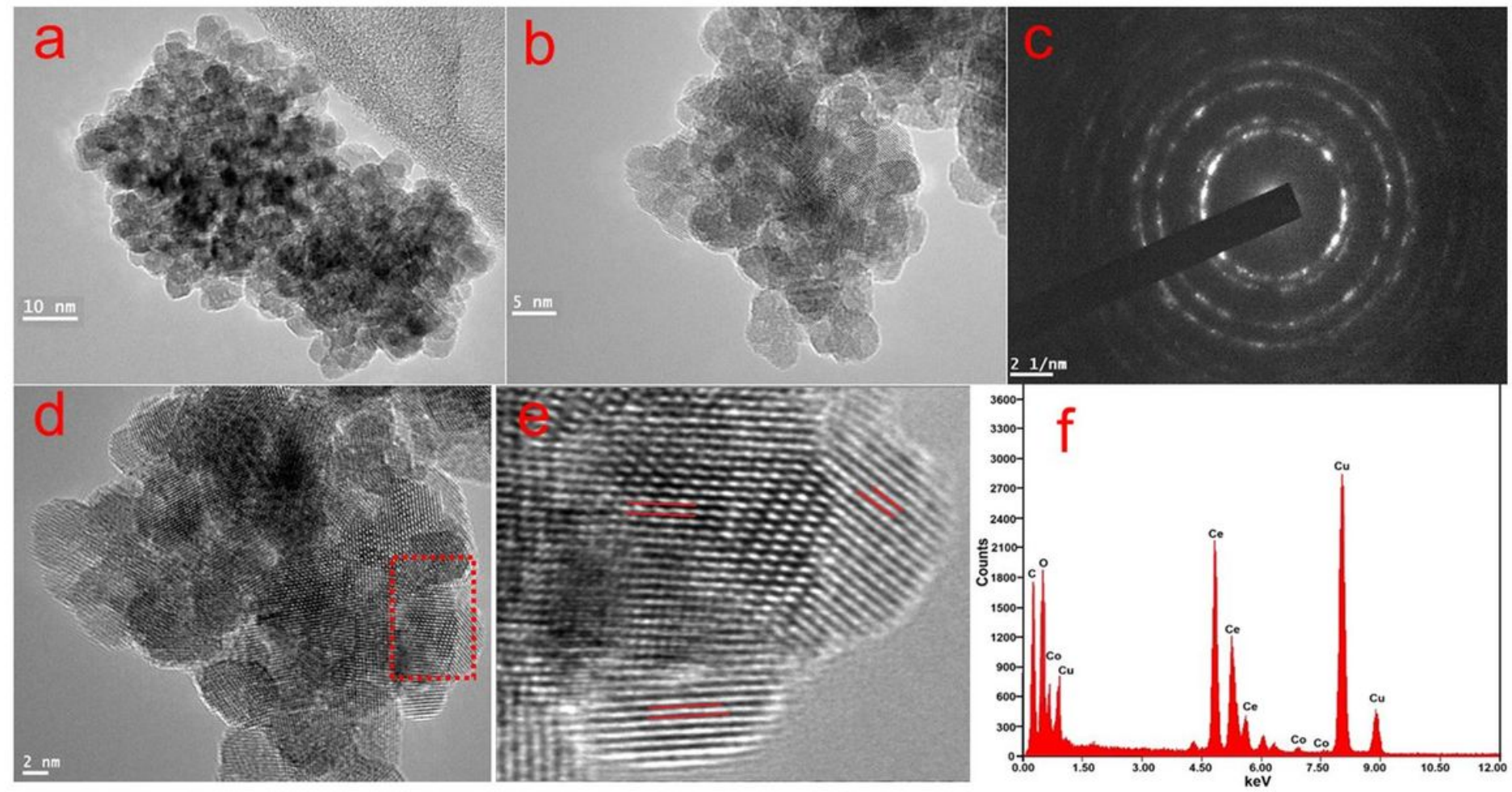

Figure 2

Field-emission transmission electron microscopic images (a) low resolution FETEM image(b) high magnifying image (c) electron diffraction pattern (d\&e) high magnifying lattice fringes image (f) EDX analysis of the as-prepared CeO2:Ag nanoparticles.
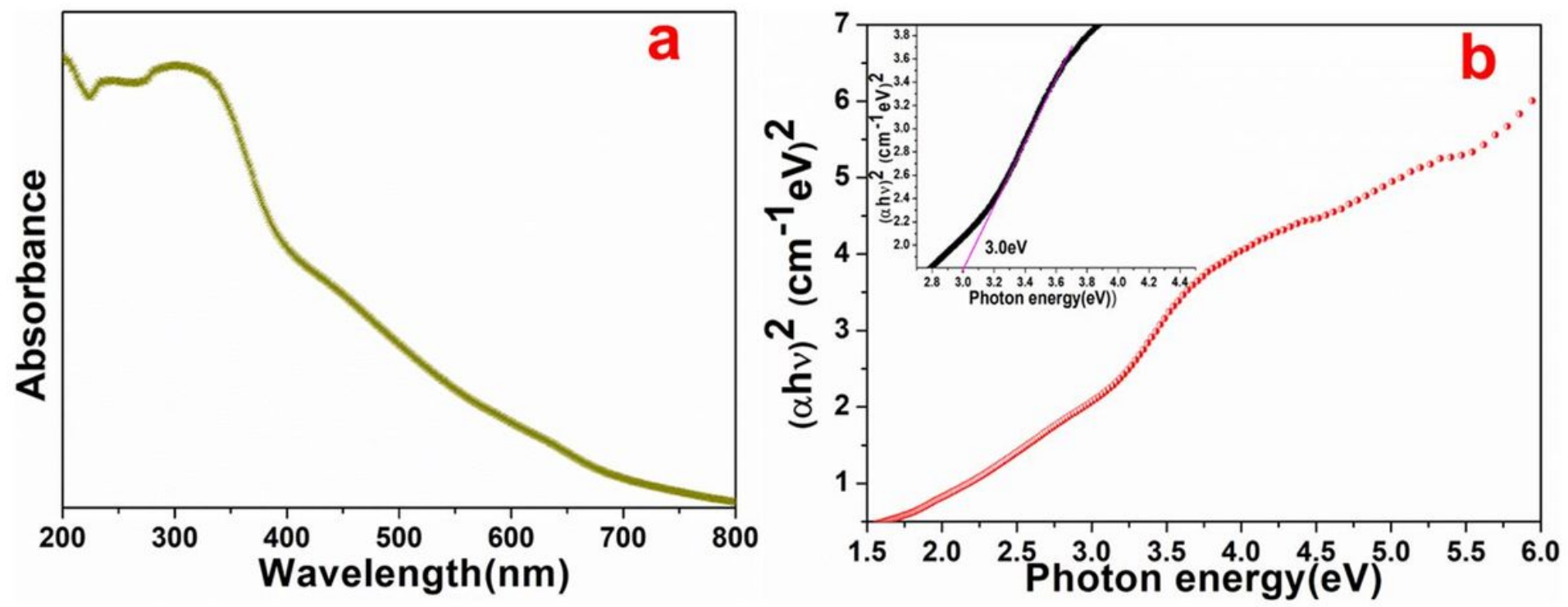

Figure 3

(a) UV/Vis absorption spectrum of CeO2:Co NPs and (b) Energy band gap, photon energy (hv) vs (ahv)2. 


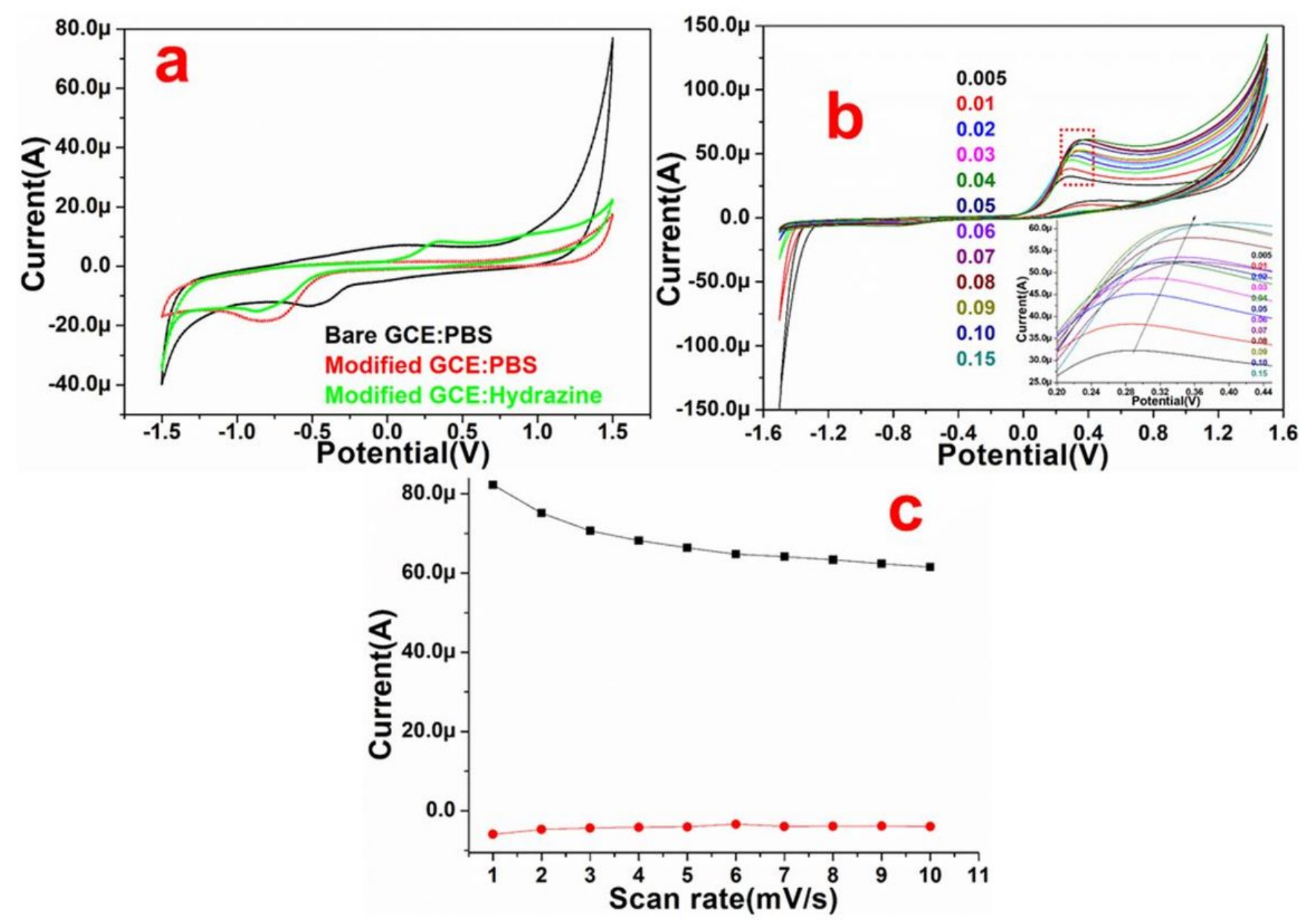

Figure 4

(a) Cyclic voltammograms of bare and modified electrodes in the absence and presence of hydrazine $(500 \mu \mathrm{M})$ in $0.01 \mathrm{M}$ phosphate buffer $(\mathrm{pH} 7.4)$ at $100 \mathrm{mVs}-1$ scan rate $(\mathrm{b} \& \mathrm{c})$. CV of CeO2:Co/GCE electrode as a function of scan rate $(5,10,20,30,40,50,60,70,80,90,100$ and $150 \mathrm{mV})$ in ascending order in PBS solution. 

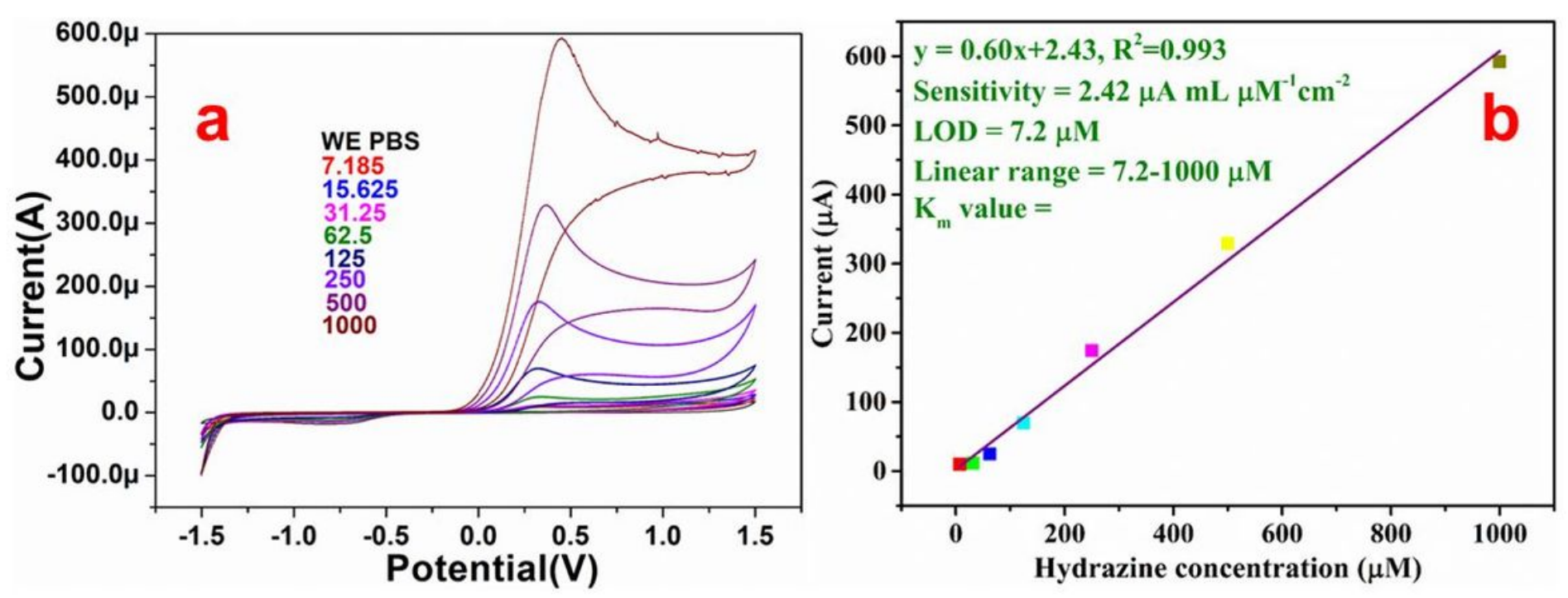

Figure 5

(a) Electrochemical responses of $\mathrm{CeO} 2 \mathrm{Co} / \mathrm{GCE}$ electrode at different concentrations of hydrazine in $0.01 \mathrm{M}$ phosphate buffer (b\&c) linearity sensitivity curve observed from CeO2:Co/GCE electrode between hydrazine concentration and current.

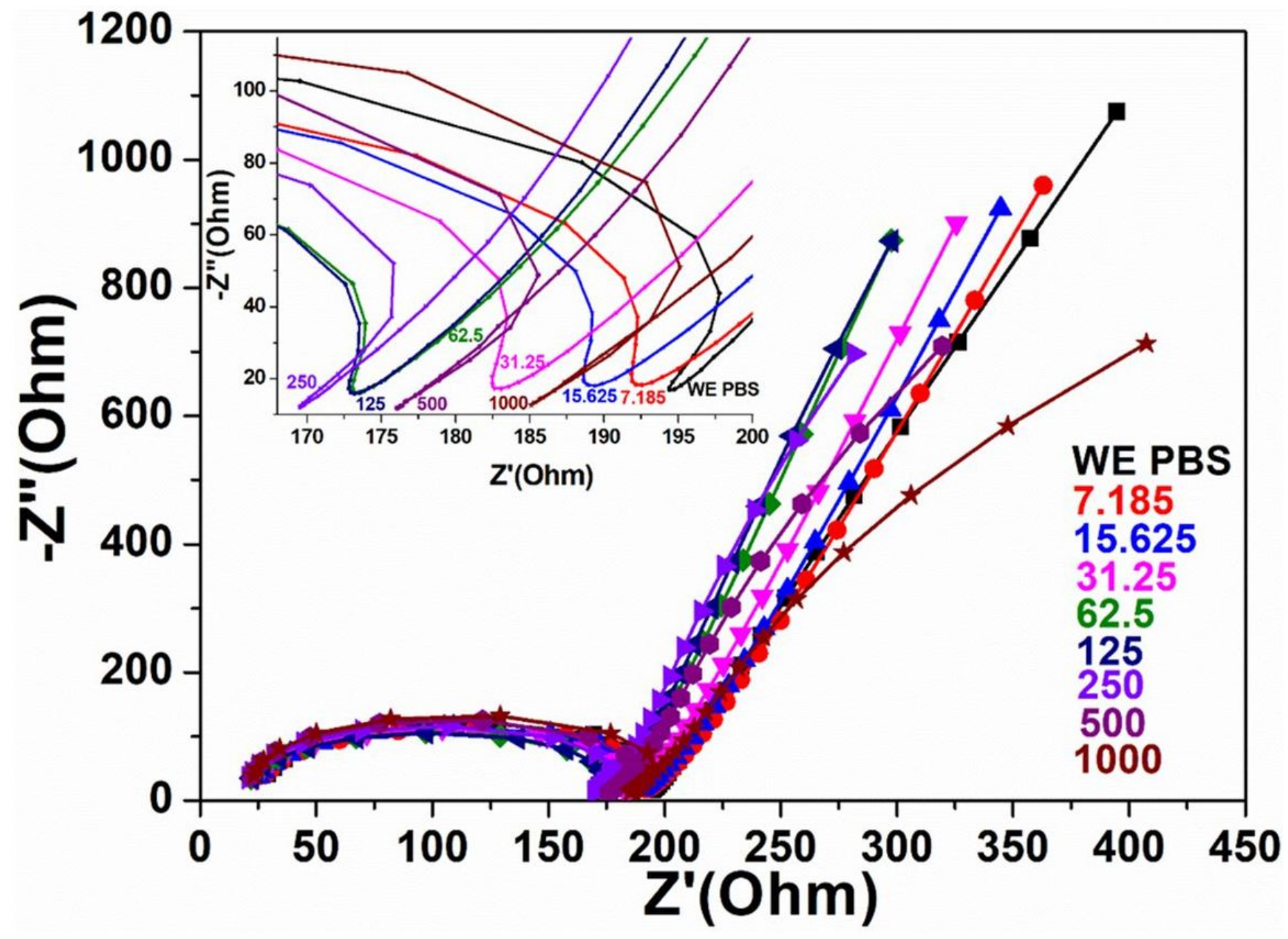


Figure 6

Electrochemical impedance spectroscopy results of $\mathrm{CeO} 2 \mathrm{Co} / \mathrm{GCE}$ electrode at different concentrations of hydrazine in $0.01 \mathrm{M}$ phosphate buffer.

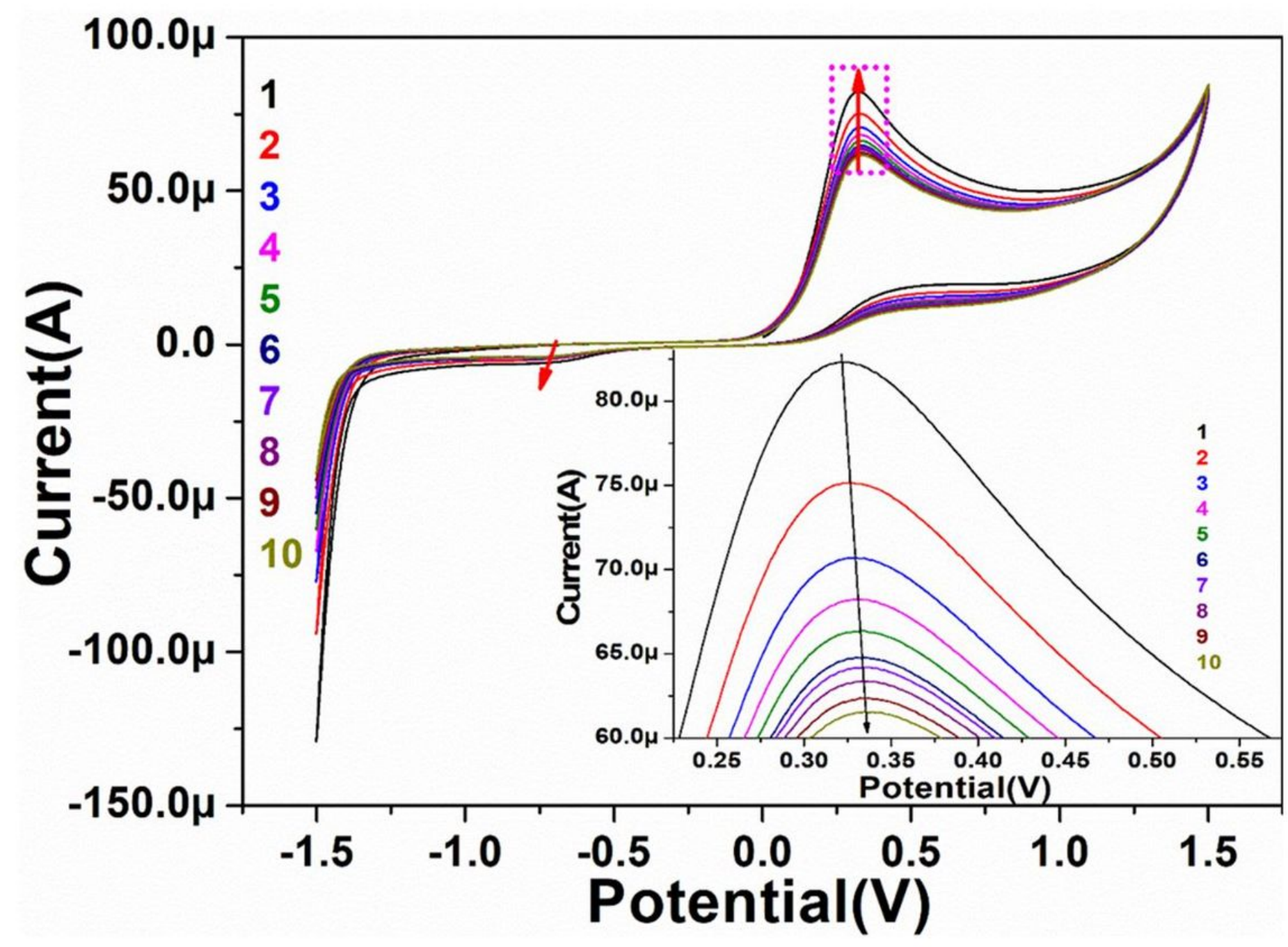

Figure 7

(a\&b) Electrochemical stability measured by $\mathrm{CV}$ at ten consecutive cycles of $\mathrm{CeO2}$ :Co/GCE electrode in presence of $500 \mu \mathrm{M}$ (hydrazine) in $0.01 \mathrm{M}$ PBS at $100 \mathrm{mV} / \mathrm{s}$. 